\title{
The orbit of a Hölder continuous path under a hyperbolic toral automorphism
}

\author{
M. C. IRWIN \\ Department of Pure Mathematics, University of Liverpool, Liverpool, L693BX, \\ England
}

(Received 4 January 1983)

\begin{abstract}
Let $f: T^{3} \rightarrow T^{3}$ be a hyperbolic toral automorphism lifting to a linear automorphism with real eigenvalues. We prove that there is a Hölder continuous path in $T^{3}$ whose orbit-closure is 1 -dimensional. This strengthens results of Hancock and Przytycki concerning continuous paths, and contrasts with results of Franks and Mañé concerning rectifiable paths.
\end{abstract}

\section{Introduction}

Let $f: T^{n} \rightarrow T^{n}$ be a hyperbolic toral automorphism. Franks proved in [1] that any compact invariant set in $T^{n}$ that contains a non-constant $C^{1}$ path also contains a torus of dimension at least two which is a coset of a subgroup of $T^{n}$. Subsequently, Mañé [5] has proved the same result for rectifiable paths. On the other hand, Hancock [2] has shown in the case $n=3$ how to construct, for all $f, C^{0}$-paths whose orbit-closures have dimension one, and Przytycki [6], using more delicate methods, has constructed such paths for all higher $n$. In his thesis, [3], Hancock asked whether the condition of Hölder continuity, which lies between continuity and rectifiability, takes paths into the Franks-Mañe or the Hancock-Przytycki camp. In this paper we prove that the latter is the case, at least for $n=3$, for certain maps $f$ and for certain values of the Hölder index. Our approach is a modification of Przytycki's. We shall deal with higher dimensional tori and invariant sets (and also more comprehensively with $n=3$ ) in another paper.

\section{Definitions and statement of the theorem}

A hyperbolic toral automorphism of $T^{n}=\mathbb{R}^{n} / \mathbb{Z}^{n}$ is a map

$$
f: T^{n} \rightarrow T^{n}
$$

that lifts to a linear automorphism

$$
L: \mathbb{R}^{n} \rightarrow \mathbb{R}^{n}
$$

with no eigenvalues of modulus 1 . Thus

(i) the matrix $A$ of $L$ has integer entries and determinant \pm 1 , and

(ii) $\mathbb{R}^{n}$ splits as the direct sum $E^{s} \oplus E^{u}$ of $L$-invariant subspaces such that all eigenvalues of $L \mid E^{s}$ and $L \mid E^{u}$ have modulus respectively $<1$ and $>1$. 
Neither the stable summand $E^{s}$ nor the unstable summand $E^{u}$ contains points of $\mathbb{Z}^{n}$ other than 0 . The cosets of $E^{s}$ and $E^{u}$ project by the standard covering map

$$
\pi: \mathbb{R}^{n} \rightarrow T^{n}
$$

onto the stable and unstable manifolds of $f$.

A map $g: X \rightarrow Y$ of metric spaces is Hölder continuous of index $\alpha(0<\alpha \leq 1)$ if, for some constant $C$,

$$
d_{Y}\left(g(x), g\left(x^{\prime}\right)\right) \leq C d_{X}\left(x, x^{\prime}\right)^{\alpha}
$$

for all $x, x^{\prime} \in X$. The case $\alpha=1$ gives Lipschitz maps. Since Lipschitz paths are rectifiable, we are only interested in the case $\alpha<1$.

When $n=3$ and $L$ is as above, the characteristic polynomial of $L$ is irreducible over $\mathbb{Z}$ and so cannot have a repeated root. Thus either $L$ or $L^{-1}$ has one eigenvalue with modulus $<1$ and two with modulus $>1$. The latter two are either real and unequal or complex conjugate. Both cases can arise, for example

$$
A=\left(\begin{array}{rrr}
1 & -1 & 0 \\
-1 & 2 & -1 \\
0 & -1 & 2
\end{array}\right), \quad A=\left(\begin{array}{rrr}
0 & 1 & 1 \\
-4 & 0 & 1 \\
1 & 1 & 1
\end{array}\right)
$$

In this paper, we restrict ourselves to the first type of automorphism. We give $T^{3}$ the flat metric.

THEOREM Let $f: T^{3} \rightarrow T^{3}$ be a hyperbolic toral automorphism lifting to a linear automorphism L. Suppose that L has real eigenvalues. Then there is a Hölder continuous path

$$
\delta:[0,1] \rightarrow T^{3}
$$

whose orbit-closure is 1-dimensional.

\section{Proof of the theorem}

We may assume, replacing $f$ by $f^{-1}$ if necessary, that $L$ has two eigenvalues $\lambda$ and $\mu$ with

$$
|\lambda|>|\mu|>1 .
$$

In fact, we may even assume that

$$
\lambda>\mu>3,
$$

for we can achieve this by replacing $f$ by some power $f^{r}$; if a path has a 1-dimensional orbit closure $\Gamma$ under $f^{\prime}$, then its orbit closure

$$
\Gamma \cup f(\Gamma) \cup \cdots \cup f^{r-1}(\Gamma)
$$

under $f$ is 1-dimensional. Our technique is to start with a linear path $\gamma_{0}$ in $E^{u}$ and, by an infinite sequence of modifications, to obtain from $\gamma_{0}$ a path $\gamma$ in $E^{u}$ such that the positive semi-orbit of $\delta=\pi \gamma$ under $f$ does not intersect a certain neighbourhood $U$ of the circle

$$
\pi\left(\mathbb{Z}^{2} \times \mathbb{R}\right)
$$

in $T^{3}$. The modified paths $\gamma_{r}$ in the sequence are all Lipschitz and our main job is to keep tabs on Lipschitz constants to ensure that the limit $\gamma$ of the sequence is 
Hölder continuous. The negative semi-orbit of $\delta$ does intersect $U$, but only near $\pi(0)$. Thus the orbit of $\delta$ is not dense in $T^{3}$. It would be quite feasible to prove directly, using Przytycki's methods, that the orbit closure of $\delta$ is 1 -dimensional. Fortunately this is not necessary, since, in the $n=3$ case, $f$ cannot have 2 - or 3-dimensional closed invariant sets apart from $T^{3}$ itself (see [4, theorem 9]).

Let

$$
G=\left(\mathbb{Z}^{2} \times \mathbb{R}\right) \cap E^{u} .
$$

We may identify $E^{u}$ with $\mathbb{R}^{2}$ under a linear isomorphism such that, in the new coordinates $(x, y)$ in $\mathbb{R}^{2}, L$ is given by

$$
L(x, y)=(\lambda x, \mu y) \text {. }
$$

We may, for convenience, suppose that the distance in $\mathbb{R}^{2}$ from 0 to the next nearest point of the lattice $G$ is greater than $2 \sqrt{2}$. It follows that if two points of $G$ are joined by a line of slope $m$ with $|m| \leq 1$ then the horizontal distance between the points is $>2$.

We now wish to describe the neighbourhood $U$ mentioned above. We fix numbers $a$ and $b$ with

$$
2 a=b<(\lambda / \mu)-1,
$$

and, for each point $(p, q) \in G$, we take a diamond-shaped neighbourhood $D_{0}(p, q)$ centred on $(p, q)$ with width $a$ and height $b$. Thus:

$$
D_{0}(p, q)=\left\{(x, y) \in \mathbb{R}^{2}: 4|x-p|+2|y-q|<b\right\} \text {. }
$$

For $r>0$, and for all $(p, q) \in f^{-r}(G)$, we define

$$
D_{r}(p, q)=f^{-r}\left(D_{0}\left(f^{r}(p, q)\right)\right)=\left\{(x, y) \in \mathbb{R}^{2}: 4 \lambda^{r}|x-p|+2 \mu^{r}|y-q|<b\right\} .
$$

We call the intersection of $D_{r}(p, q)$ and the line $x=p$, the vertical core of $D_{r}(p, q)$. We further insist that $b$ is small enough for the following condition to hold:

$\left(2_{0}\right)$ if a line of slope $m$ with $|m| \leq 1$ intersects distinct diamonds $D_{0}(p, q)$ and $D_{0}\left(p^{\prime}, q^{\prime}\right)$ then

$$
\left|p-p^{\prime}\right|>2 \text {. }
$$

Notice that this implies, for all $r \geq 0$,

(2r) if a line of slope $m$ with $|m| \leq \lambda^{r} / \mu^{r}$ intersects distinct diamonds $D_{r}(p, q)$ and $D_{r}\left(p^{\prime}, q^{\prime}\right)$ then

$$
\left|p-p^{\prime}\right|>2 / \lambda^{r}
$$

Finally, let $\tilde{D_{r}}(p, q)$ be $D_{r}(p, q)$ with $a$ and $b$ replaced by fixed numbers $\tilde{a}$ and $\tilde{b}$ satisfying

$$
2 \tilde{a}=\tilde{b}<b(\mu-3) /(\mu-1)
$$

and let $D$ be the union of $\tilde{D}_{0}(p, q)$ for all $(p, q) \in G$. Identifying $E^{u}$ with a subset of $\mathbb{R}^{3}$ once more, let $V$ be the vertical cylinder generated by $D$ (that is to say, $p^{-1}(p(D))$, where $p$ is vertical projection of $\mathbb{R}^{3}$ onto the plane $z=0$ ) and let $U=\pi(V)$.

Let $I=[0,1]$. We define maps

$$
\gamma_{r}: I \rightarrow \mathbb{R}^{2} \quad(r \geq 0)
$$


inductively, starting with $\gamma_{0}(t)=(t, 0)$. We assume that $\gamma_{r-1}$ is defined $(r \geq 1)$ and is the graph of a piecewise linear function

$$
g_{r-1}: I \rightarrow \mathbb{R}
$$

with Lipschitz constant

$$
\text { Lip } g_{r-1} \leq \lambda^{r-1} / \mu^{r-1} \text {. }
$$

Thus if $\gamma_{r-1}(I)$ intersects any diamond $D_{r-1}(p, q)$, it intersects its vertical core. Also, by $\left(2 r_{r-1}\right)$, if $\gamma_{r-1}(I)$ intersects two such vertical cores, the horizontal distance between them is greater than $2 / \lambda^{r-1}$. Suppose that the intersections of $\gamma_{r-1}(I)$ with such vertical cores are at

$$
\gamma_{r-1}\left(t_{i}\right), \quad 1 \leq i \leq m,
$$

and that $\gamma_{r-1}\left(t_{i}\right)$ is distance $b_{i}$ below the top of the vertical core (so that $b_{i}<b / \mu^{r-1}$ ). We define $\gamma_{r}$ by

$$
\gamma_{r}(t)= \begin{cases}\gamma_{r-1}(t) & \text { if }\left|t-t_{i}\right| \geq 1 / \lambda^{r-1} \quad \text { for all } i, \\ \left(t, g_{r-1}(t)+(1-|u|) b_{i}\right) & \text { if } t=t_{i}+u / \lambda^{r-1},-1 \leq u \leq 1, \quad \text { for some } i .\end{cases}
$$

We write $\gamma_{r}(t)=\left(t, g_{r}(t)\right)$. Notice that, for all $t \in I$,

$$
0 \leq g_{r}(t)-g_{r-1}(t) \leq b / \mu^{r-1} \text {. }
$$

Also

$$
\begin{aligned}
\operatorname{Lip} g_{r} & \leq \operatorname{Lip} g_{r-1}+\operatorname{Lip}\left(g_{r}-g_{r-1}\right) \\
& \leq \lambda^{r-1} / \mu^{r-1}+b\left(\lambda^{r-1} / \mu^{r-1}\right) \\
& \leq \lambda^{r} / \mu^{r}
\end{aligned}
$$

by (1), as required for the induction. By (4), the sequence $\left(\gamma_{r}\right)$ converges to a path $\gamma$ which is the graph of a function $g: I \rightarrow \mathbb{R}$. Moreover, since by (4), for all $s \geq r$ and all $t \in I$,

$$
0 \leq g_{s}(t)-g_{r}(t) \leq b /\left[\mu^{r-1}(\mu-1)\right]
$$

and by (3),

$$
b / 2 \mu^{r-1}-\tilde{b} / 2 \mu^{r-1}>b /\left[\mu^{r-1}(\mu-1)\right]
$$

the fact that $\gamma_{r}(I)$ does not intersect any $D_{r-1}(p, q)$ implies that $\gamma_{s}(I)$ does not intersect any $\tilde{D}_{r}(p, q)$, for any $r \geq 0$.

Now note that, for all $r>0$ and $1 / \lambda^{r}<\left|t-t^{\prime}\right|<1 / \lambda^{r-1}$,

$$
\begin{aligned}
\left|g(t)-g\left(t^{\prime}\right)\right| & \leq\left|g_{r}(t)-g_{r}\left(t^{\prime}\right)\right|+\left|\left(g(t)-g_{r}(t)\right)-\left(g\left(t^{\prime}\right)-g_{r}\left(t^{\prime}\right)\right)\right| \\
& \leq\left(\lambda^{r} / \mu^{r}\right)\left|t-t^{\prime}\right|+b /\left[\mu^{r-1}(\mu-1)\right] \quad(b y(5) \text { and }(6)) \\
& \leq\left(\lambda^{r} / \mu^{r}\right)(1+b \mu /(\mu-1))\left|t-t^{\prime}\right| .
\end{aligned}
$$

Write

$$
C=(\lambda / \mu)(1+b \mu /(\mu-1))
$$

Then (7) says that

$$
\left(\left|t-t^{\prime}\right|,\left|g(t)-g\left(t^{\prime}\right)\right|\right)
$$


lies beneath the straight line segment joining $\left(1 / \lambda^{r},(\mu / \lambda) C / \mu^{r}\right)$ to $\left(1 / \lambda^{r-1}, C / \mu^{r-1}\right)$. This is below the segment of the curve

$$
y=C x^{\log \mu / \log \lambda}
$$

joining $\left(1 / \lambda^{r}, C / \mu^{r}\right)$ to $\left(1 / \lambda^{r-1}, C / \mu^{r-1}\right)$, since $\mu / \lambda<1$ and the curve is concave downwards. This shows that $g$ is Hölder continuous with constant $C$ and index $\log \mu / \log \lambda$, and hence that $\gamma$ is Hölder continuous with constant $\sqrt{1+C^{2}}$ and index $\log \mu / \log \lambda$.

Finally note that, by construction, images of $\gamma$ under positive iterates of $L$ avoid $V$ entirely, while images under negative iterates intersect $V$ in $\tilde{D}_{0}(0,0)$ only. Thus, as explained above, the image of $\delta=\pi \gamma$ has 1-dimensional orbit-closure.

\section{REFERENCES}

[1] J. Franks. Invariant sets of hyperbolic toral automorphisms. Amer. J. Math. 99 (1977), 1089-1095.

[2] S. G. Hancock. Construction of invariant sets for Anosov diffeomorphisms. J. London Math. Soc. (2) 18 (1978), 339-348.

[3] S. G. Hancock. Invariant sets of Anosov diffeomorphisms. Thesis, Warwick University, 1979.

[4] M. W. Hirsch. On invariant subsets of hyperbolic sets. In Essays on Topology and Related Topics. Springer-Verlag, 1970.

[5] R. Mañé. Orbits of paths under hyperbolic toral automorphisms. Proc. Amer. Math. Soc. 73 (1979), 121-125.

[6] F. Przytycki. Construction of invariant sets for Anosov diffeomorphisms and hyperbolic attractors. Studia Math. 68 (1980), 199-213. 